\title{
SHP2 inhibition reduces leukemogenesis in models of combined genetic and epigenetic mutations
}

\author{
Ruchi Pandey, ${ }^{1}$ Baskar Ramdas, ${ }^{1}$ Changlin Wan, ${ }^{2}$ George Sandusky, ${ }^{3}$ Morvarid Mohseni, ${ }^{4}$ Chi Zhang, ${ }^{5}$ and Reuben Kapur ${ }^{1}$ \\ 'Herman B Wells Center for Pediatric Research, ${ }^{2}$ Department of Electrical and Computer Engineering, and ${ }^{3}$ Department of Pathology, Indiana University School of Medicine, Indianapolis, Indiana, USA. ${ }^{4}$ Oncology \\ Disease Area, Novartis Institute of Biomedical Research, Cambridge, Massachusetts, USA. ${ }^{5}$ Department of Medical and Molecular Genetics, Indiana University School of Medicine, Indianapolis, Indiana, USA.
}

\begin{abstract}
In patients with acute myeloid leukemia (AML), $10 \%$ to $30 \%$ with the normal karyotype express mutations in regulators of DNA methylation, such as TET2 or DNMT3A, in conjunction with activating mutation in the receptor tyrosine kinase FLT3. These patients have a poor prognosis because they do not respond well to established therapies. Here, utilizing mouse models of AML that recapitulate cardinal features of the human disease and bear a combination of loss-of-function mutations in either Tet2 or Dnmt3a along with expression of Flt3 ${ }^{1 T D}$, we show that inhibition of the protein tyrosine phosphatase SHP2, which is essential for cytokine receptor signaling (including FLT3), by the small molecule allosteric inhibitor SHP099 impairs growth and induces differentiation of leukemic cells without impacting normal hematopoietic cells. We also show that SHP099 normalizes the gene expression program associated with increased cell proliferation and self-renewal in leukemic cells by downregulating the Myc signature. Our results provide a new and more effective target for treating a subset of patients with AML who bear a combination of genetic and epigenetic mutations.
\end{abstract}

\section{Introduction}

Although acute myeloid leukemia (AML) is the second most common form of leukemia, the 5-year survival rate has remained dismally low $(27 \%)$. The prognosis is worse for older patients and for those patients who harbor a combination of certain genetic and epigenetic mutations (1). Mutations in genes regulating DNA methylation such as TET2 and DNMT3A are common in preleukemic cells and often evolve into AML with the acquisition of additional mutations such as $F L T 3^{I T D}$ (2). In mice, combining loss-of-function mutation(s) in genes regulating DNA methylation (Tet2 or Dnmt3a) with Flt3 $3^{\text {TT }}$ transforms preleukemic cells into fully penetrant and lethal AML (3-5). These models of AML not only phenocopy the mutational spectrum and clinical features observed in patients with AML but also simulate resistance to standard therapies. Similarly, leukemic Tet2 ${ }^{-1-F l t 3^{I T D}}$ mice show an initial reduction in leukemic blasts followed by relapse due to drug-resistant leukemic cells (3). The therapy-resistant cells in AML patients tend to have a stem cell-like gene signature profile (6). The leukemia-initiating potential in these mouse models lies in the most primitive stem cell compartment. Both conventional chemotherapy and FLT3 ${ }^{\text {ITD }}$-targeted therapies such as AC220 (Quizartinib) or crenolanib do not impact the function of leukemia stem cells (LSCs) as a single agent (3). However, AC220 induces a partial normalization of LSCs when used in combination with 5-azacytidine $(7,8)$. Although the FDA recently approved gilteritinib, a FLT3 ${ }^{\mathrm{ITD}}$ specific inhibitor, for treatment of refractory AML (9), it hasn't been tested in similar models of AML. Thus, there is a need for effective treatment strategies for these subsets of AML.

Conflict of interest: MM is an employee of Novartis.

Copyright: ๔ 2019, American Society for Clinical Investigation.

Submitted: May 21, 2019; Accepted: September 3, 2019; Published: November 4, 2019.

Reference information: J Clin Invest. 2019;129(12):5468-5473.

https://doi.org/10.1172/JCl130520.
Use of clinically relevant mouse models of AML allows for validation of targets that are likely to be effective in the clinic. We hypothesized that targeting SHP2, a protein tyrosine phosphatase, will be a more effective strategy than current treatment modalities for treating AMLs that possess a combination of genetic and epigenetic mutations. Not only is SHP2 associated with positive signaling downstream of FLT3 ${ }^{\text {ITD }}$ (10), it is also involved in regulating signaling via multiple cytokine receptors and is likely to be involved in driving the growth and survival of preleukemic cells bearing epigenetic mutations (11). So far, clinical application of SHP2 inhibitors has been limited due to lack of specificity and efficacy. Recently, an allosteric inhibitor, SHP099, has been described that overcomes the lack of specificity associated with previous generation inhibitors of SHP2 that target the active site of the enzyme (12). SHP099 was shown to inhibit FLT3 ${ }^{\text {ITD }}$ AML in a xenotransplant model but detailed characterization of its effects on multiple differentiation stages of leukemic cells, or gene expression was not done (12). Here, we evaluated the therapeutic efficacy of SHP099 in 2 clinically relevant mouse models of AML carrying a combination of genetic $\left(F l t 3^{I T D}\right)$ and epigenetic (Tet2 or Dnmt3a) mutations in leukemic cells.

\section{Results and Discussion}

SHP099 attenuates leukemogenesis driven by Tet2 ${ }^{-/-}$Flt $3^{I T D}$. To evaluate the effect of targeting SHP2 in leukemic cells, BM cells carrying Tet2 $2^{-/}$Flt $3^{I T D}$ mutations on a CD $45.2^{+}$background were transplanted in F1 mice $\left(\mathrm{CD} 45.1^{+} \mathrm{CD} 45.2^{+}\right)$with normal WT cells on a congenic CD $45.1^{+}$background. This allowed us to examine the impact of SHP099 in parallel on both normal (WT: CD45.1 $1^{+}$and leukemic $\left(\mathrm{CD} 45.2^{+}\right)$cells (Supplemental Figure 1B; supplemental material available online with this article; https://doi.org/10.1172/ JCI130520DS1). All mice developed features consistent with leukemia, including increased peripheral blood counts (WBC), which 
A

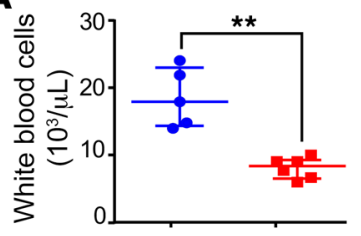

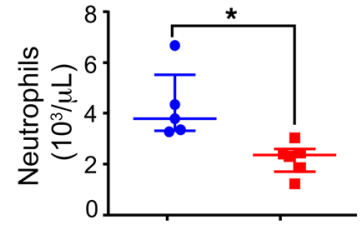

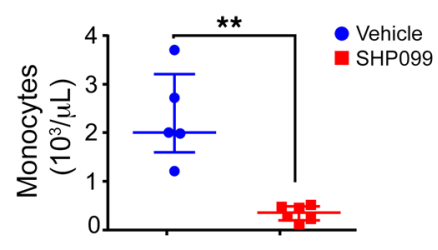

B

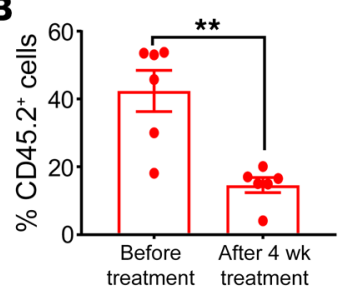

C

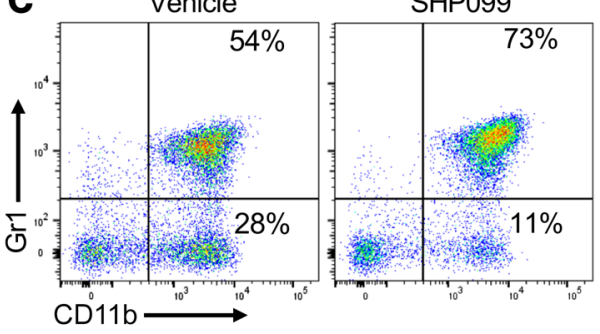

G

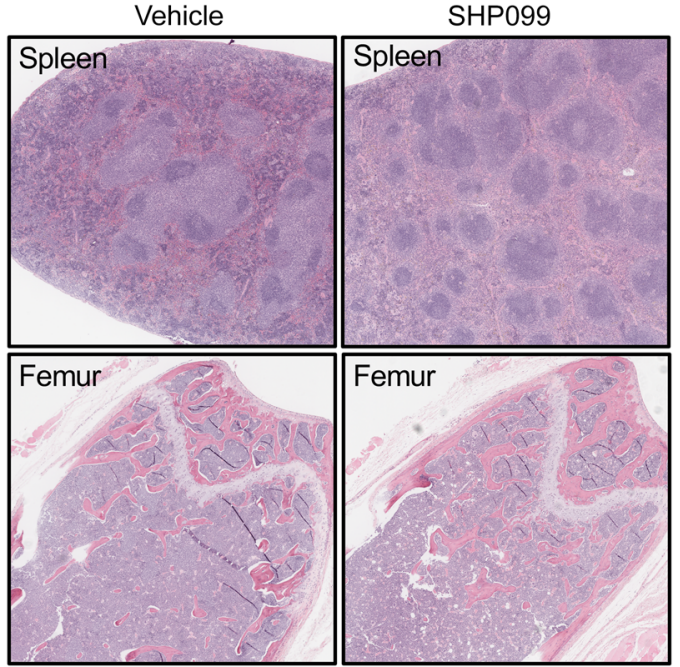

D Vehicle

SHP099

Gated on CD45.1-

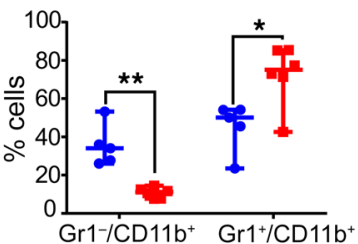

$\mathbf{E}$

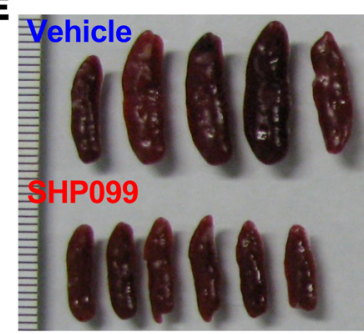

H
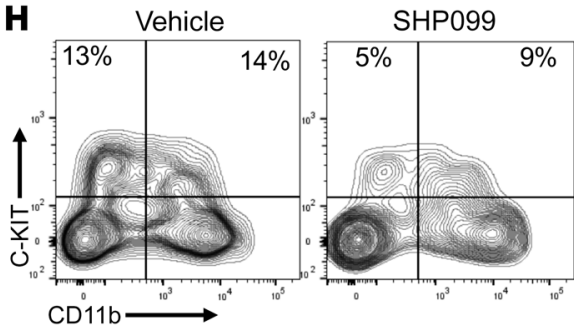

I

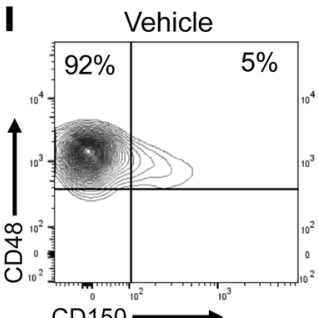

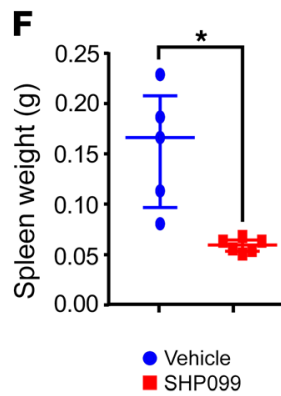

$\mathbf{J}$
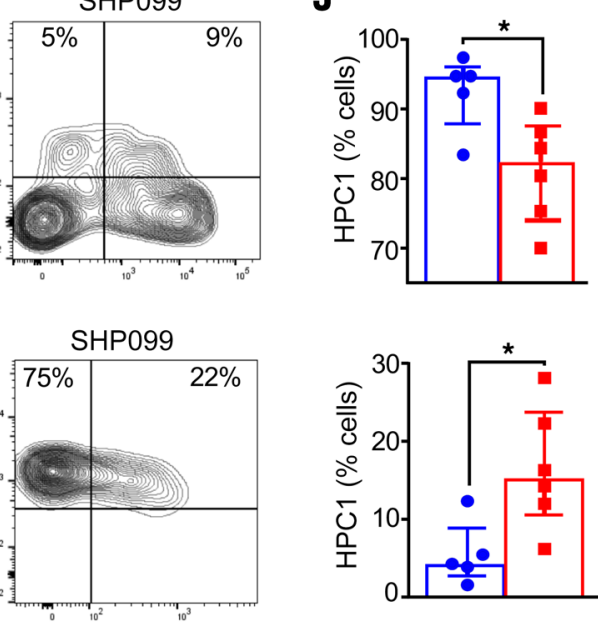

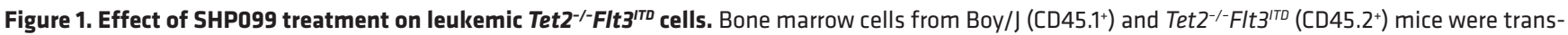
planted in irradiated F1 (CD45.1+CD45.2+) mice followed by treatment with vehicle $(n=5)$ or SHP099 $(n=6)$ for 4 weeks. (A) Number of WBCs, neutrophils, and monocytes in the PB. (B) Percentage CD45.2+CD45.1 cells in PB. (C) Representative flow cytometric profiles of Gr1 and CD11b expression in PB cells gated on CD45.1- (D) Quantification of Gr1-CD11 b+ and Gr1+CD11b+ cells within CD45.1- gate. (E) Image of spleens from treated mice. (F) Quantification of spleen weight. (G) Representative H\&E-stained images from spleen and femur of treated mice, acquired at $\times 20$. (H) Representative flow plots of CD11b and c-KIT expression in CD45.1 - gated spleen cells. (I) Representative flow plots of CD48 and CD150 expression in CD45.1 -lin-Sca1+C-KIT+ (leukemic LSK) cells. (J) Quantification of HPC1 (CD48+CD150) and HPC2 (CD48+CD150+) cells therein. Data points are values from individual mice in each group from 1 of the 2 representative experiments. Median value for each group is indicated with interquartile range. ${ }^{*} P<0.05,{ }^{*} P<0.01$; Student's $t$ test with Welch's correction for unequal variance.

were markedly reduced within 1 week of initiating the treatment with SHPO99 (data not shown). The specificity of SHP099 for inhibiting the growth of leukemic $\left(\mathrm{CD} 45.2^{+}\right)$cells but not normal WT (CD45.1 $1^{+}$cells was confirmed by examining the ratio of CD 45.2 $2^{+}$versus CD $45.1^{+}$cells in SHP099-treated mice. SHP099 treatment resulted in significant reduction in the frequency of CD45.2 $2^{+}$leukemic cells (Figure 1B), which was associated with a concomitant increase in the frequency of CD $45.1^{+}$WT cells (Supplemental Figure 1B) while the relative percentages of $\mathrm{CD} 45.2^{+}$ leukemic cells were unchanged during the course of treatment in the vehicle-treated group (Supplemental Figure 1C). By the end of 4 weeks of treatment, the WBC counts were normalized and there was a concomitant reduction in the number of monocytes and neutrophils in peripheral blood (PB) (Figure 1A). SHP099 treatment had no significant impact on other blood parameters such as lymphocytes, red blood cells (RBC), hemoglobin, and platelet numbers (Supplemental Figure 1A). Importantly, drug treatment not only reduced the number of leukemic myeloid cells but also reversed the monocytic skewing (increase in $\mathrm{Gr}^{-} \mathrm{CD} 11 \mathrm{~b}^{+}$cells) observed in leukemic cells to a more differentiated neutrophilic $\left(\mathrm{Gr}^{+} \mathrm{CD} 11 \mathrm{~b}^{+}\right)$phenotype (Figure 1, C and D).

SHP099 normalized splenomegaly associated with $\mathrm{Tet}^{2-1}$ Flt $3^{I T D}$ leukemia (Figure 1, E and F). Similar to the observations in the PB, SHP099 treatment significantly reduced the percentage of leukemic cells $\left(C D 45.2^{+}\right)$in the spleen (Supplemental Figure 1D), further confirming that SHP099 has preferential activity against leu- 
A

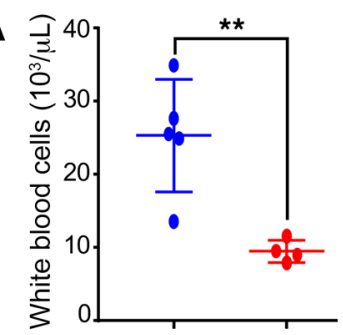

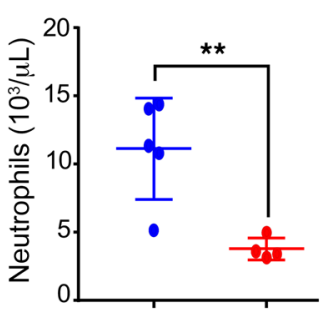

D

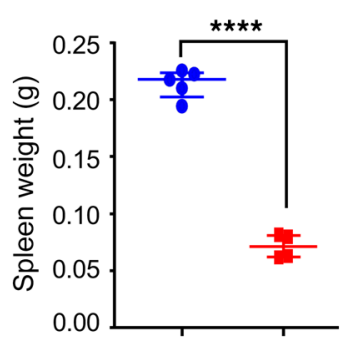

G

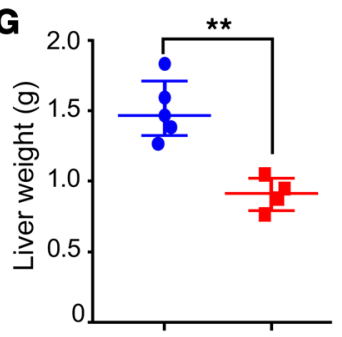

B

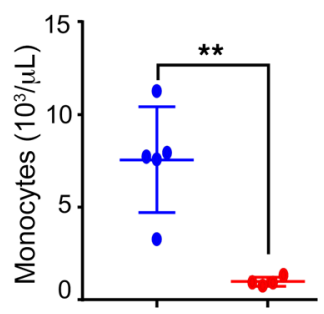

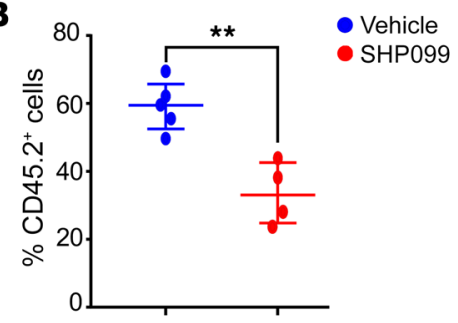

C

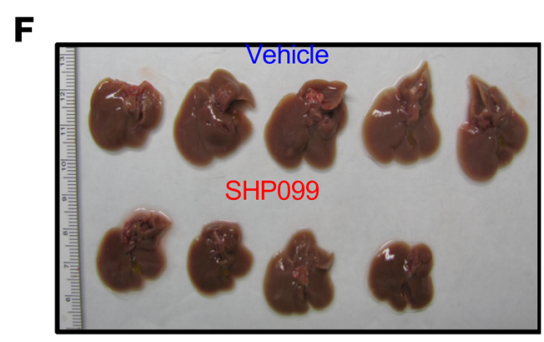

E

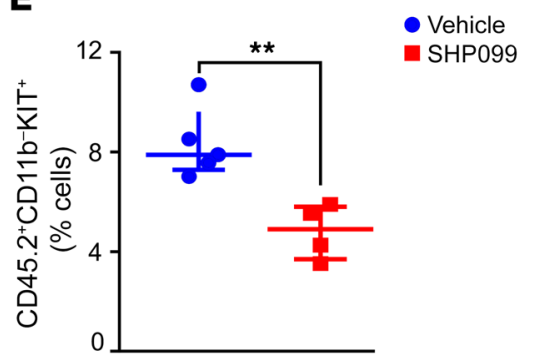

H

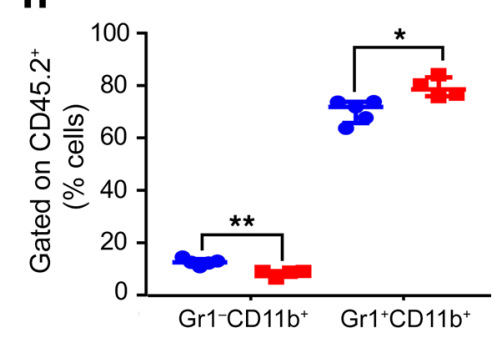

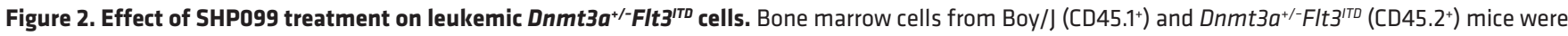
transplanted in irradiated Boy/J (CD45.1 $1^{+}$mice and treated with vehicle $(n=5)$ or SHP099 $(n=4)$. (A) WBC, neutrophil, and monocyte counts in PB. (B) Percentage of CD45.2 $2^{+}$cells in PB. (C) Image of spleen. (D) Quantification of spleen weight. (E) Percent CD11b-c-KIT ${ }^{+}$cells in spleen within CD45.2 ${ }^{+}$gate. (F) Image of liver. (G) Quantification of liver weight. (H) Percentage of Gr1-CD11 b+ and Gr1+CD11b+ cells in the BM in CD45.2+ gate. Data points shown are values from individual mice in each group. Median value for each group is indicated with the interquartile range. ${ }^{*} P<0.05,{ }^{* *} P<0.01,{ }^{* * *} P<0.0001 ;$ Student's $t$ test with Welch's correction for unequal variance.

kemic cells compared with normal cells. In the vehicle-treated mice, the normal splenic architecture was lost due to increased infiltration of immature myeloid leukemic cells, leading to diffused germinal centers (Figure 1G). Drug treatment partially restored the structural organization of the spleen and increased the number of distinct germinal centers in the treated group (Figure 1G). Extramedullary hematopoiesis was also significantly reduced by SHP099, as evidenced by reduction in the frequency of immature CD11b ${ }^{-} \mathrm{KIT}^{+}$ leukemic cells (Figure 1H and Supplemental Figure 1E). Similarly, histological examination of the femur from the vehicle-treated mice showed hypercellularity and loss of normal vascular channels due to an increase in myeloid blasts (Figure 1G). Drug treatment partially restored the vascular sinuses and myeloid-to-erythroid ratio in these mice (Figure $1 \mathrm{G}$ ). In the $\mathrm{Tet}^{-1-} \mathrm{Flt3}^{I T D}$ leukemia model, the disease initiation potential is present within the lin-Scal $^{+} \mathrm{CKIT}^{+}$ CD $48^{+} \mathrm{CD} 150^{-}$cells (also called HPC1 cells) (3). The normal heterogeneity within the lin $^{-} \mathrm{Sca}^{+}{ }^{+} \mathrm{CKIT}^{+}$(LSK) compartment is almost completely lost in this model, and it is made up of mono-phenotypic HPC1 cells (3). Our analysis showed that drug treatment reduced the frequency of both leukemic immature lin $^{-}$and LSK cells (Supplemental Figure 2, A-C), and induced differentiation in the leu- kemia-initiating HPC1 cells to HPC2 cells $\left(\mathrm{CD} 48^{+} \mathrm{CD} 150^{+}\right)$(Figure 1, I and J). HPC2 cells have increased multi-lineage reconstitution potential as compared with HPC1 cells. These results are in striking contrast to treatment of leukemic mice carrying mutant Tet2-Flt $3^{I T D}$ cells with a FLT3 ${ }^{\text {ITD }}$-specific inhibitor, AC220 (3). While AC220 was successful in reducing the peripheral leukemic burden, it had no impact on the stem cell compartment (3). Interestingly, none of the other drugs that have been tested in this model of AML have demonstrated a similar level of selectivity toward leukemic cells or ability to induce differentiation in LSCs when used as a single agent, except for 5-azacytidine $(3,7,8)$. However, 8 treatment cycles of 21 days each with 5-azacytidine were required to induce differentiation in the LSC compartment (7), whereas our results show that an approximately 30-day treatment with SHP099 is able to induce a similar level of differentiation in LSCs (Figure 1, I and J). Thus, in addition to specifically repressing the growth of leukemic cells, targeting SHP2 with SHP099 is sufficient to induce differentiation of LSCs in the BM and terminal differentiation of leukemic myeloid cells in the periphery.

SHP099 attenuates leukemogenesis driven by Dnmt $3 a^{+/-F l t 33^{I T D}}$ cells. Loss-of-function mutations in DNMT3A, another regulator 
A
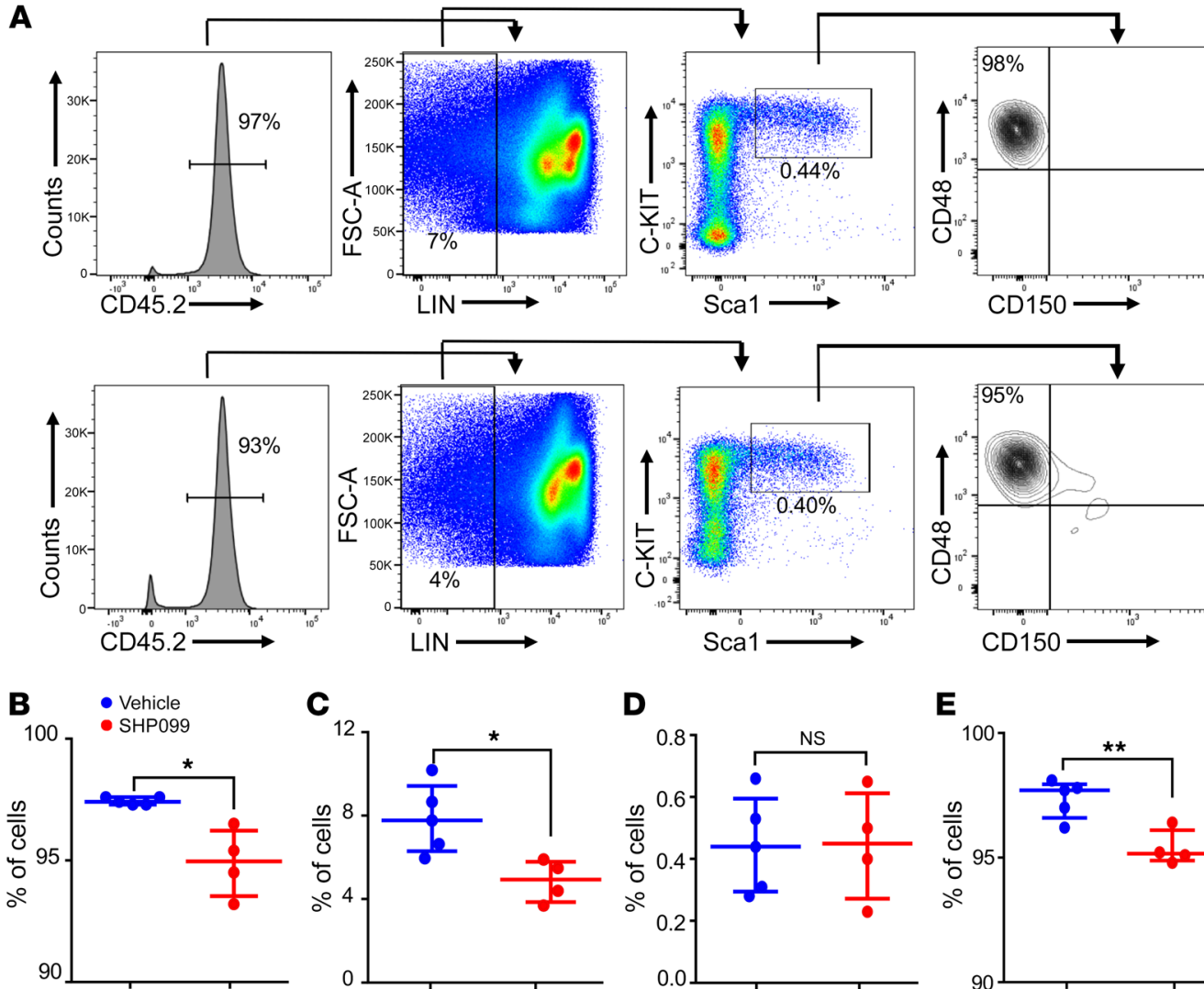

D

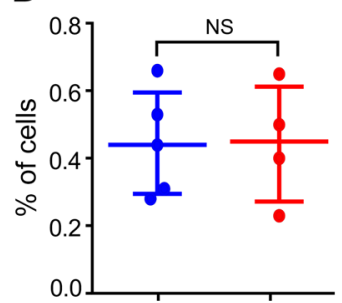

E

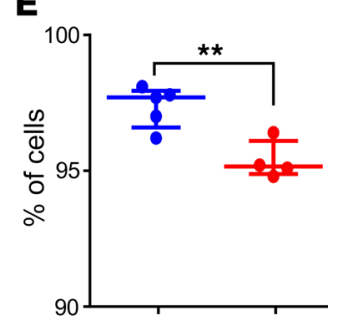

Figure 3. Effect of SHPO9g treatment on bone marrow stem cells

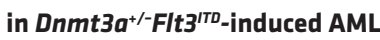
model. Analysis of bone marrow cells from mice transplanted with Dnmt3a+/-Flt3 ${ }^{\text {ITD }}$ (CD45.2+) and Boy/J (CD45.1+) cells and treated with vehicle or SHPO99 as in

Figure 2. (A) Representative flow plots showing the analysis strategy (top, vehicle-treated mice; bottom, SHP099-treated mice). Quantification of (B) percentage of CD45.2+ cells, (C) percentage of lin ${ }^{-}$ cells, (D) percentage of LSK cells in the leukemic (CD45.2+) compartment and (E) percentage of HPC1 (lin-Sca1+KIT+CD48+CD150-) within the CD45.2+LSK gate. Data points shown are values from individual mice in each group. Median value for each group is indicated with the interquartile range. ${ }^{*} P<0.05$, ${ }^{* *} P<0.01$; Student's $t$ test with Welch's correction for unequal variance. of DNA methylation, are also commonly found in association with FLT3 ${ }^{\text {ITD }}$ in AML patients. Loss of a single Dnmt3a allele in combination with Flt $3^{I T D}$ leads to transformation of the preleukemic and MPN phenotypes observed in the single mutant mice into a fully penetrant and lethal AML $(4,5)$. To determine if attenuating effects of SHP099 observed in Tet $2^{-/}-F_{t} 3^{I T D}$-driven AMLs can also be extended to Dnmt $3 a^{+/-F l t 3} 3^{I T D}$-driven AML, bone marrow cells carrying Dnmt3a $a^{+/} F l t 3^{I T D}$ mutations (CD $45.2^{+}$) were transplanted in congenic mice $\left(C D 45.1^{+}\right)$along with normal congenic CD $45.1^{+}$

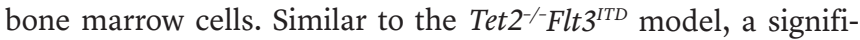
cant decrease in peripheral WBC counts along with a decrease in the percentage of leukemic CD $45.2^{+}$cells was observed in these mice within 1 week of SHP099 treatment (data not shown). Drug treatment was stopped after 2 weeks, as some mice showed signs of weakness, but was reinitiated after a 10-day recovery period for an additional 10 days, for a total of 25 days. The group of

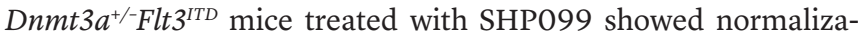
tion of WBC numbers, with the most significant correction once again observed in the myeloid compartment (i.e., monocytes and neutrophils) (Figure 2A). Similar to our previous results utilizing the Tet2 ${ }^{-/-}$Flt $3^{I T D}$ AML model, the effects seen in the Dnmt $3 a^{+/-}$ Flt $3^{I T D}$ model of AML were due to specific targeting of leukemic cells by SHP099, as evidenced by a decrease in the relative percentage of CD $45.2^{+}$leukemic cells (Figure 2B). Treatment with SHP099 ameliorated splenomegaly (Figure 2, C and D) and hepatomegaly (Figure 2, F and G) associated with $\mathrm{Dnmt3}^{+/-} \mathrm{Flt} 3^{I T D_{-}}$ induced leukemia. The normalization of spleen size by treatment with SHP099 was accompanied by a reduction in immature $\mathrm{Kit}^{+}$cells in the spleen (Figure $2 \mathrm{E}$ ). Similar to the effects of
SHP099 in AML driven by Tet2 $2^{-/}$Flt3ITD mutations, inhibition of SHP2 by SHP099 in Dnmt3a $a^{+-}$Flt3ITD cells reversed monocytic skewing and induced differentiation of the terminal myeloid cells $\left(\mathrm{GR} 1^{+} \mathrm{CD} 11 \mathrm{~b}^{+}\right.$cells; Figure $\left.2 \mathrm{H}\right)$. Treatment of mice carrying Dnmt $3 a^{+/-F l t} 3^{I T D}$ mutant cells with SHP099 also reduced the bone marrow colonization by leukemic cells, as evidenced by a decrease in the percentage of CD $45.2^{+}$cells (Figure $3, \mathrm{~A}$ and $\mathrm{B}$ ). The reduction in leukemic cells by SHP099 was accompanied by a reduction in immature cells (lin ${ }^{-}$cells; Figure 3, A and C). Though there was no significant difference in the percentage of LSK cells between the vehicle and SHP099 treated groups (Figure 3, A and D); the LSCs showed increased differentiation upon SHP099 treatment (Figure 3, A and E). These results demonstrate that inhibition of SHP2 by SHP099 is effective in attenuating leukemogenesis driven by Flt3 $3^{I T D}$ in combination with either $\mathrm{Tet}^{-/-}$or $\mathrm{Dnm} t 3 \mathrm{a}^{+/-}$, by possibly similar mechanisms.

SHP099 treatment normalizes aberrant gene expression in Tet2--Flt $3^{\text {ITD }}$ cells. To elucidate the mechanism of action of SHP099 on leukemic cells, lineage-depleted cells were isolated from the bone marrow of $\mathrm{Tet}^{-/-} \mathrm{Flt} 3^{\text {ITD }}$ mice and cultured in the presence of cytokines. These cells were subsequently treated with SHP099 or vehicle for 24 hours and RNA-seq was performed on samples from 3 independent cultures established from individual mice. For comparison of gene expression profiles, lineage-depleted WT cells were similarly isolated and cultured. Differential gene expression and pathway enrichment analysis of the RNA-seq data from these cells showed significant changes in the expression of genes involved in regulating cell proliferation, including in the MYC pathway (Figure 4, A and D). Specifically, the cell prolifer- 
A

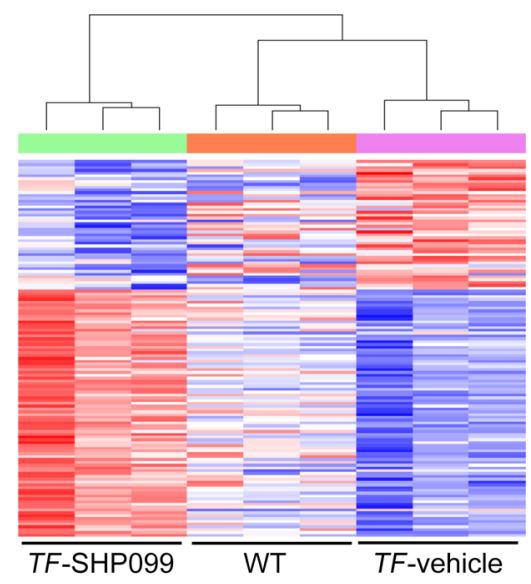

B

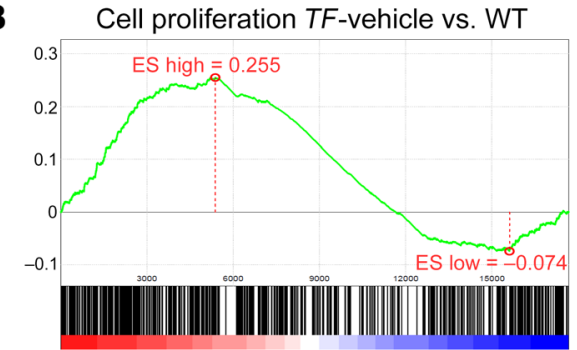

C

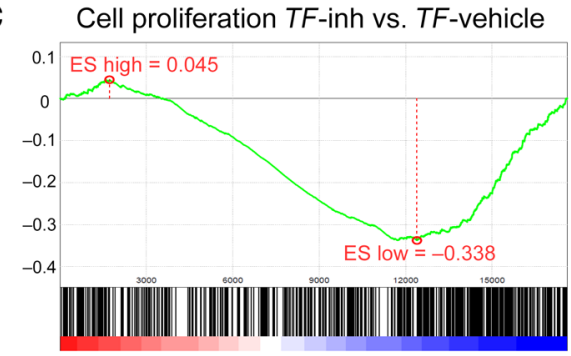

D

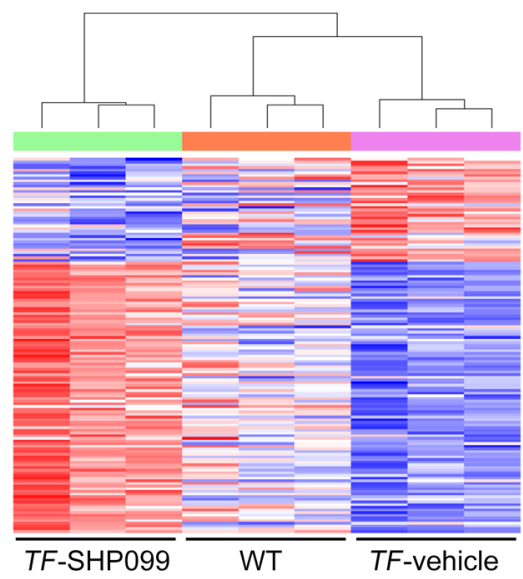

E

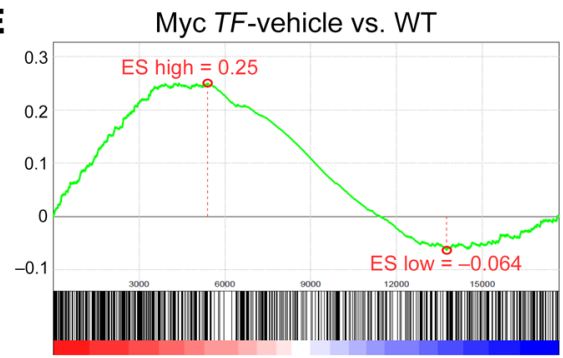

F

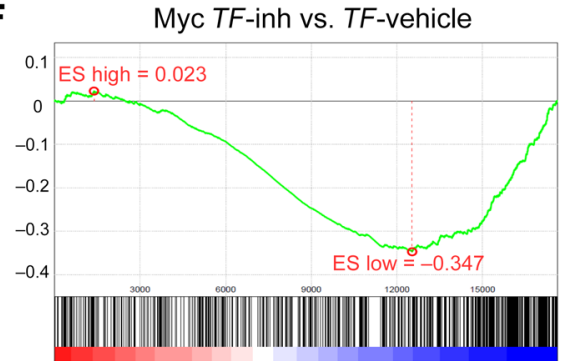

Color key

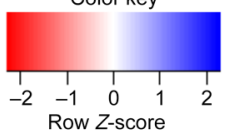

Figure 4. Effect of SHPOgg treatment on gene expression in Tet2 ${ }^{-1-F I t 3^{1 T D}}$ (TF) cells. Lineage-depleted bone marrow cells from individual mice $(n=3)$ were expanded in cytokine cocktail (SCF, IL-3, and IL-6) and treated with SHPO99 or vehicle for 24 hours. RNA was isolated and subjected to RNA-seq analysis. (A) Heatmap of differentially expressed genes regulating proliferation. (B) GSEA of genes regulating proliferation in TF vehicle-treated vs. WT cells. (c) GSEA analysis of genes regulating proliferation in TF vehicle- vs. SHPOg9-treated cells. (D) Heatmap of differentially expressed genes in the MYC pathway. (E) GSEA analysis of MYC signature in TF vehicle vs. WT treatment. (F) GSEA analysis of MYC signature in TF vehiclevs. SHP099-treated cells. (C) Expression of genes that regulate self-renewal. Individual values from 3 biological replicates are shown. Columns indicate the median value and error bars denote the range. The color code for the heatmap is indicated.
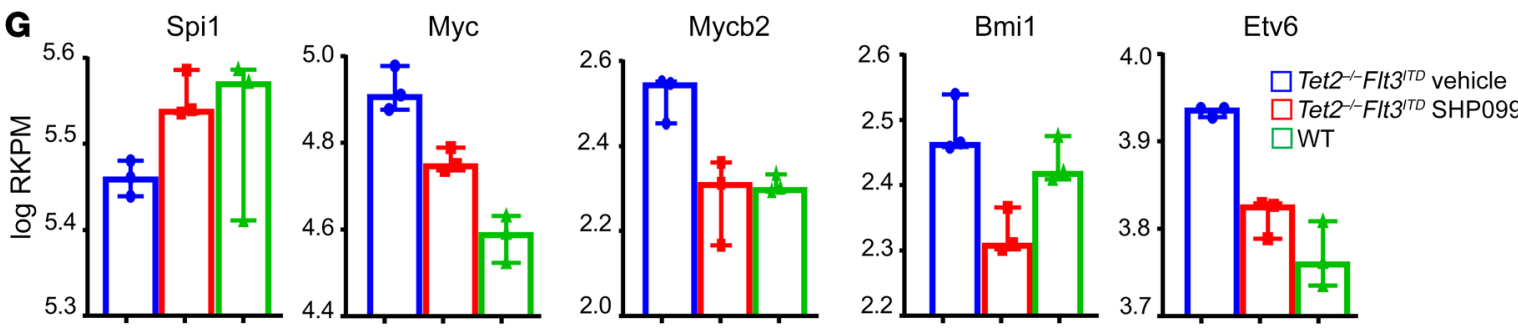

ation pathway was significantly upregulated in vehicle-treated cells from Tet $2^{--}-F l t 3^{I T D}$ mice vs. cells derived from WT mice ( $P=5$ $\times 10^{-3}$; Figure $4 \mathrm{~B}$ ). The upregulation was reversed upon treatment of these cells with SHPO99 $\left(P=8 \times 10^{-15}\right.$; Figure 4C). Similarly, the MYC-regulated genes were significantly enriched in leukemic cells from Tet2 $2^{-F l t 3} 3^{I T D}$ mice vs. cells derived from WT mice (Figure 4E). Enrichment of the MYC-regulated genes was also reversed upon treatment with SHP099 (Figure 4F). MYC-regulated genes play an important role in inhibiting the differentiation of leukemic myeloid cells and development of AML (13), and their repression by SHP099 would contribute to its antileukemic activity. Furthermore, several genes associated with self-renewal that were upregulated in Tet2 $2^{--F l t 3^{I T D}}$ cells, including $M y c, M y c b p 2$, Etv6, and Bmi1, were largely normalized to WT levels upon
SHP099 treatment (Figure 4G). In contrast, genes such as Spi1 (Pu.1) that limit self-renewal, induce myeloid cell differentiation, and are downregulated in Tet2 ${ }^{-}-F_{t} 3^{I T D}$ cells compared with WT cells, were upregulated in response to SHPO99 treatment (Figure $4 \mathrm{G}$ ), which is consistent with the in vivo effects of SHP099 on LSCs and myeloid cell differentiation.

In conclusion, we have demonstrated that SHP099, an allosteric SHP2 inhibitor, is effective as a single agent in 2 clinically relevant models of AML. This confirms the antileukemic activity of SHP099 previously reported in a xenotransplant model with a FLT3 ${ }^{\text {ITD }}$ AML patient sample (12). While the other coexisting mutations in the patient sample were not defined, we here show the efficacy of targeting SHP2 in models carrying mutations in epigenetic regulators that are known to confer primary resis- 
tance to FLT3-specific inhibitors (8). The gene expression and phenotypic analysis suggest that therapeutic activity of SHP099 is through induction of differentiation in primitive progenitor stem cells and committed myeloid leukemia cells. The decrease in the percentage of immature cells suggests that SHP099 regulates their expansion through inhibition of proliferation. Our results with SHP099 in 2 models of AML driven by a combination of mutations regulating DNA methylation and cellular signaling provide improvement over studies utilizing single agents such as AC220, AG221, or 5-azacytdine in similar models of AML $(3,7)$. SHP099 appears to be more effective than AC220, suggesting that inhibition of SHP2 blocks pathways other than those activated by FLT3 ${ }^{\text {ITD }}$. Some of these mutations are known to alter the cytokine milieu of the bone marrow (14), and since SHP2 plays an important role in cytokine signaling (11), SHP099 likely inhibits pathways that may not be susceptible to inhibition by AC220. Our results provide an additional target in AML that merits further probing and has the potential to provide relief in these subsets of patients with AML.

\section{Methods}

Mice. Tet2 ${ }^{--}$, Flt3 ${ }^{I T D}$, Dnmt3afl/fl (15-17), and Mx1-Cre mice on CD45.2 background were intercrossed to generate Tet $2^{-/}-F l t 3^{I T D}$ and Dnmt $3 a^{+/ f l}$ Flt $3^{I T D} \mathrm{M} x 1 \mathrm{Cr} e^{+}$mice. Dnmt $3 a^{+f l} \mathrm{Flt} 3^{I T D} \mathrm{M} x 1 \mathrm{Cr} e^{+}$mice were treated with poly IC to induce deletion of the floxed allele and hereafter are referred

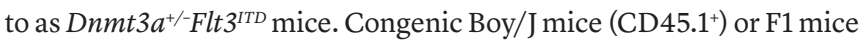
$\left(\mathrm{CD} 45.1^{+} \mathrm{CD} 45.2^{+}\right)$were procured from The Jackson Laboratory or the Indiana University School of Medicine (IUSM) core facility.

RNA sequencing, differential gene expression, and pathway enrichment analysis. The RNA samples were processed and sequenced at the Center for Medical Genomics, IUSM (details in Supplemental Methods). The data were normalized using the trimmed mean of $\mathrm{M}$ values method and genes with read count per million greater than 0.5 in more than 3 of the samples were kept. Differentially expressed genes were tested using DESeq2 with a FDR less than 0.05 as the significant cutoff (18). Pathway enrichment analyses were conducted by hypergeometric tests against mouse Gene Ontology and MsigDB v6 canonical pathways, with $P$ less than 0.001 as the significant cutoff (19). For selected pathways, GSEA enrichment score and ssGSEA sample-wise enrichment score were used for visualization (19). The raw data have been submitted to the NCBI GEO database (accession number GSE134843).

Statistics. Mice were randomly assigned to the treatment groups and none of the data points were excluded from analysis. Two-tailed Student's $t$ test with Welch's correction for unequal variance was used to determine significant differences $(P<0.05)$ using Prism 6 software.

Study approval. The mouse protocol was approved by the IACUC and all experiments were conducted at the Laboratory Animal Resource Center as per the protocol.

\section{Author contributions}

$\mathrm{RP}$ and RK conceived the study and designed experiments. RP and $\mathrm{BR}$ did the experiments and analyzed data. CW and CZ analyzed RNA-seq data. GS did histopathological analysis. MM provided critical reagents. RP, RK, BR, and CZ wrote the manuscript. All authors read and approved the manuscript.

\section{Acknowledgments}

This work was supported by NIH grants R01CA173852, R01CA134777, R01HL146137, and R01HL140961, and Riley Children's Foundation (to RK). CZ and RP were supported by funds from the Grace M. Showalter Trust.

Address correspondence to: Ruchi Pandey or Reuben Kapur, R4, 1044 W. Walnut Street, Indianapolis, Indiana 46202, USA. Phone: 317.274.0303; Email: pandeyru@iupui.edu (RP). Phone: 317.274.4658; Email: rkapur@iupui.edu (RK).
1. Liersch R, Müller-Tidow C, Berdel WE, Krug U. Prognostic factors for acute myeloid leukaemia in adults--biological significance and clinical use. Br J Haematol. 2014;165(1):17-38.

2. Bowman RL, Busque L, Levine RL. Clonal hematopoiesis and evolution to hematopoietic malignancies. Cell Stem Cell. 2018;22(2):157-170.

3. Shih AH, et al. Mutational cooperativity linked to combinatorial epigenetic gain of function in acute myeloid leukemia. Cancer Cell. 2015;27(4):502-515.

4. Meyer SE, et al. DNMT3A haploinsufficiency transforms FLT3ITD myeloproliferative disease into a rapid, spontaneous, and fully penetrant acute myeloid leukemia. Cancer Discov. 2016;6(5):501-515.

5. Yang L, et al. DNMT3A loss drives enhancer hypomethylation in FLT3-ITD-associated leukemias. Cancer Cell. 2016;29(6):922-934.

6. Shlush LI, et al. Tracing the origins of relapse in acute myeloid leukaemia to stem cells. Nature. 2017;547(7661):104-108.

7. Shih $\mathrm{AH}$, et al. Combination targeted therapy to disrupt aberrant oncogenic signaling and reverse epigenetic dysfunction in IDH2- and TET2mutant acute myeloid leukemia. Cancer Discov. 2017;7(5):494-505.

8. Zhang $\mathrm{H}$, et al. Clinical resistance to crenolanib in acute myeloid leukemia due to diverse molecular mechanisms. Nat Commun. 2019;10(1):244.

9. Dhillon S. Gilteritinib: First global approval. Drugs. 2019;79(3):331-339.

10. Pandey R, Saxena M, Kapur R. Role of SHP2 in hematopoiesis and leukemogenesis. Curr Opin Hematol. 2017;24(4):307-313.

11. Cai Z, et al. Inhibition of inflammatory signaling in Tet 2 mutant preleukemic cells mitigates stress-induced abnormalities and clonal hematopoiesis. Cell Stem Cell. 2018;23(6):833-849.e5.

12. Chen YN, et al. Allosteric inhibition of SHP2 phosphatase inhibits cancers driven by receptor tyrosine kinases. Nature. 2016;535(7610):148-152.

13. Delgado MD, León J. Myc roles in hematopoiesis and leukemia. Genes Cancer. 2010;1(6):605-616.

14. Binder S, Luciano M, Horejs-Hoeck J. The cyto- kine network in acute myeloid leukemia (AML): A focus on pro- and anti-inflammatory mediators. Cytokine Growth Factor Rev. 2018;43:8-15.

15. Li Z, et al. Deletion of Tet 2 in mice leads to dysregulated hematopoietic stem cells and subsequent development of myeloid malignancies. Blood. 2011;118(17):4509-4518.

16. Lee BH, et al. FLT3 mutations confer enhanced proliferation and survival properties to multipotent progenitors in a murine model of chronic myelomonocytic leukemia. Cancer Cell. 2007;12(4):367-380.

17. Yu Q, et al. DNA methyltransferase 3 a limits the expression of interleukin-13 in T helper 2 cells and allergic airway inflammation. Proc Natl Acad Sci U S A. 2012;109(2):541-546.

18. Love MI, Huber W, Anders S. Moderated estimation of fold change and dispersion for RNA-seq data with DESeq2. Genome Biol. 2014;15(12):550.

19. Subramanian A, et al. Gene set enrichment analysis: a knowledge-based approach for interpreting genome-wide expression profiles. Proc Natl Acad Sci U S A. 2005;102(43):15545-15550. 